\title{
Drowning hazard with deep brain stimulation: case report
}

\author{
Omar K. Bangash, MBBS, ${ }^{1,2}$ Megan Thorburn, PGDipN, ${ }^{2}$ Jimena Garcia-Vega, BAppSc (Physio), ${ }^{1,3}$ \\ Susan Walters, BAppSc (Physio), ${ }^{4}$ Rick Stell, MBBS, FRACP, ${ }^{4,5}$ Sergio E. Starkstein, MD, PhD, ${ }^{6}$ \\ and Christopher R. P. Lind, FRACS ${ }^{1,2}$
}

\begin{abstract}
${ }^{1}$ School of Surgery, University of Western Australia; ${ }^{2}$ Neurosurgical Service of Western Australia, Sir Charles Gairdner Hospital; ${ }^{3}$ Department of Physiotherapy, Sir Charles Gairdner Hospital; ${ }^{4}$ Western Australian Neuroscience Research Institute; ${ }^{5}$ Department of Neurology, Sir Charles Gairdner Hospital; and ${ }^{6}$ Neuropsychiatry Unit, School of Psychiatry and Clinical Neurosciences, University of Western Australia, Perth, Australia
\end{abstract}

\begin{abstract}
The caudal zona incerta target within the posterior subthalamic area is an investigational site for deep brain stimulation (DBS) in Parkinson disease (PD) and tremor. The authors report on a patient with tremor-predominant PD who, despite excellent tremor control and an otherwise normal neurological examination, exhibited profound difficulty swimming during stimulation. Over the last 20 years, anecdotal reports have been received of 3 other patients with PD who underwent thalamic or pallidal lesioning or DBS surgery performed at the authors' center and subsequently drowned. It may be that DBS puts patients at risk for drowning by specifically impairing their ability to swim. Until this finding can be further examined in larger cohorts, patients should be warned to swim under close supervision soon after DBS surgery.

http://thejns.org/doi/abs/10.3171/2015.5.JNS15589
\end{abstract}

KEY WORDS deep brain stimulation; posterior subthalamic area; drowning; swimming; Parkinson disease; functional neurosurgery

$\mathrm{D}$ EEP brain stimulation (DBS) for medically refractory Parkinson disease (PD) and essential tremor has become established neurosurgical practice in helping to alleviate troublesome motor symptoms. Level 1 evidence supports targeting of the subthalamic nucleus (STN) and the internal segment of the globus pallidus for PD. ${ }^{1,2,10,11}$ A promising refinement of the STN target is centered on the caudal zona incerta (cZI) at the border of the posteromedial STN within the posterior subthalamic area (PSA). We have used a slightly more anterior cZI target than that initially described by Plaha et al. because of concerns about long-term effects on gait (S. Gill, personal communication, 2007). ${ }^{7}$

An avid swimmer with PD, who was enrolled in a Phase II clinical trial (ClinicalTrials.gov, no. NCT01945567) comparing clinical outcome with DBS within dorsal and ventral subsegments of the PSA, lost the ability to swim with stimulation. This was despite having excellent resolution of motor symptoms. Remarkably, on examination the patient had no neurological deficits as determined by an experienced neurologist (R.S.); he could swim without dif- ficulty with the stimulator turned off, but had severe difficulty swimming with it turned on.

This report raises concern about the possibility of drowning with PSA DBS, which requires immediate attention. The ability of traditional neurological examination to identify such complex motor deficits may require reevaluation. Whether similar effects are associated with DBS at other targets is uncertain: we have received 3 additional anecdotal reports of deaths by drowning in patients from our center undergoing hybrid lesioning and DBS at the ventrointermediate nucleus of the thalamus and pallidum for PD over the past 20 years. We hypothesize that DBS stimulation of the PSA may induce task-specific disturbance of learned motor function.

\section{Case Report}

History and Examination

A right-handed, 68-year-old man presented with a 5-year history of medication-refractory, tremor-predominant PD. The tremor had begun in his right hand and progressed

ABBREVIATIONS cZI = caudal zona incerta; DBS = deep brain stimulation; MDS-UPDRS-III = Movement Disorder Society-Unified Parkinson's Disease Rating Scale Part III (motor section); PD = Parkinson disease; PSA = posterior subthalamic area; STN = subthalamic nucleus.

SUBMITTED March 14, 2015. ACCEPTED May 14, 2015.

INCLUDE WHEN CITING Published online November 13, 2015; DOI: 10.3171/2015.5.JNS15589. 
to involve the entire right upper and lower limbs. Prior to being seen in the clinic, he had also begun experiencing left upper-limb symptoms. The tremor had both postural and kinetic components and was worse with stress and under observation. The patient had difficulty eating, writing, and performing other dexterous hand movements bilaterally. He had severely limited his social interactions due to embarrassment associated with his tremor. There was moderate rigidity, bradykinesia, and akinesia. There was no dystonia or motor fluctuations, nor were there medication-induced dyskinesias. He had no cognitive or psychiatric symptoms or signs on structured interviews. Dopaminergic medication trials were associated with intolerable side effects, including severe nausea and vomiting. The patient had a medical history of ischemic heart disease with no active symptoms, mild rheumatoid arthritis, and sciatica that was well managed nonoperatively.

\section{Operation}

After discussing treatment options, the decision was made to perform PSA DBS as part of a local clinical trial. The PSA was targeted bilaterally under general anesthesia, using the MRI-directed implantable guide tube technique as a staged procedure. ${ }^{6,9}$ Carbothane proxy markers of electrode position (NeuroGuide, Renishaw Plc) were placed stereotactically to the cZI targets, and a 3-T intraoperative MRI study was performed after administration of a second general anesthetic agent. Once position was confirmed at the posteromedial border of the STN (Fig. 1), the stylets were replaced with quadripolar DBS electrodes (Model 3389, Medtronic, Inc.).

As part of the clinical trial protocol, the patient was randomly assigned to either dorsal or ventral stimulation for 3 months each, with frequency of stimulation constant at $130 \mathrm{~Hz}$, pulse width constant at $60 \mu \mathrm{sec}$, and amplitude set up to a maximum of $3 \mathrm{~mA}$. These two settings were followed by a 6-month period of empirical programming by a movement disorder neurologist. During the empirical programming period the patient experienced almost complete resolution of tremor, with reduction in bradykinesia and rigidity and no change in gait on neurological examination. Final program settings and active electrode positions are shown in Table 1 and Fig. 1, respectively.

\section{Postoperative Course}

The therapeutic effect was characterized by a reduction in the patient's score on the Movement Disorder SocietyUnified Parkinson's Disease Rating Scale (MDS-UPDRS) Part III (motor section) from a postoperative stimulationoff score of 36 to a score of 2 with stimulation on, based

\section{TABLE 1. Settings for DBS electrodes*}

\begin{tabular}{llllc}
\hline \multicolumn{1}{c}{ Program Setting } & \multicolumn{2}{c}{ Lt Brain } & \multicolumn{2}{c}{ Rt Brain } \\
\hline Interleaving settings & A & B & A & B \\
\hline Cathode (electrode contact) & 1 & 2 & 9 & 10 \\
\hline Voltage (V) & 3.5 & 3.5 & 1.5 & 3.0 \\
\hline
\end{tabular}

* A bilateral interleaving program of monopolar stimulation programs $A$ and $B$ was used. Each cathode is numbered according to the Medtronic Model 3389 lead contact assignment. The device's case served as the anode. Stimulation frequency was $130 \mathrm{~Hz}$ and pulse width was $60 \mu \mathrm{sec}$. on empirical parameters (Table 2). Assessments were performed without dopaminergic medications due to intolerable side effects. In addition to excellent tremor control, the patient's other symptoms of PD improved, and he was very satisfied with the result. On stable stimulation settings more than several months after surgery, the patient felt confident in resuming his swimming at his home pool. Before surgery he had been a regular lap swimmer. On first returning to the swimming pool, he quickly realized he could not propel himself adequately and that he required assistance to get to safety. The patient reported the event immediately to our team.

\section{Supervised Swimming Assessment and Outcome}

The patient was advised to avoid swimming, and an appointment was made for a pool-based assessment under physiotherapy, nursing, and research supervision. This was conducted and filmed in the controlled environment of the hospital physiotherapy swimming pool. The patient's swimming was assessed first with DBS switched on. The stimulator was then switched off and the patient rested for 30 minutes. The patient's swimming was then reassessed with DBS switched off. No dopaminergic medications were taken during assessments.

On stimulation the patient was unable to propel himself forward or float adequately unassisted. There was a lack of synchronization of upper and lower limbs, and an unusual rotation of the trunk during the following strokes: freestyle, backstroke, and breaststroke. The patient tended to jackknife and fail to move forward during attempted freestyle. An attempt was made to attenuate upper-limb involvement with a flutter board, which did not improve performance. Freestyle was improved somewhat by immobilizing the lower limbs. This was achieved by asking the patient to swim while gripping Styrofoam cylinders between his legs.

After withdrawal of stimulation, the patient's ability to swim improved remarkably. He was able to swim freestyle, backstroke, and breaststroke in a well-coordinated fashion. Footage of the patient swimming freestyle with and without stimulation can be seen in Video 1.

VIDEO 1. DBS ON: The patient is observed attempting to swim freestyle. Within the first few strokes there is a marked inability to propel himself or float. The patient's movements lack synchronization between upper and lower limbs, and there is an unusual rotation of the trunk with each stroke. DBS OFF: The same freestyle swimming task is attempted after the DBS device has been switched off for 30 minutes. The patient is seen swimming effectively and floating unassisted. Increased coordination between upper and lower limbs as well as greater stability through the trunk is observed with each stroke. Copyright Omar K. Bangash. Published with permission. Click here to view.

\section{Discussion}

This case raises the possibility that patients undergoing DBS of the PSA for tremor-predominant PD may be at risk for drowning. With stimulation switched on, this patient developed an objective worsening of swimming ability, which appeared to particularly reduce control of his lower limbs and posture in the water. At a minimum this risk needs to be considered for DBS of the PSA for 

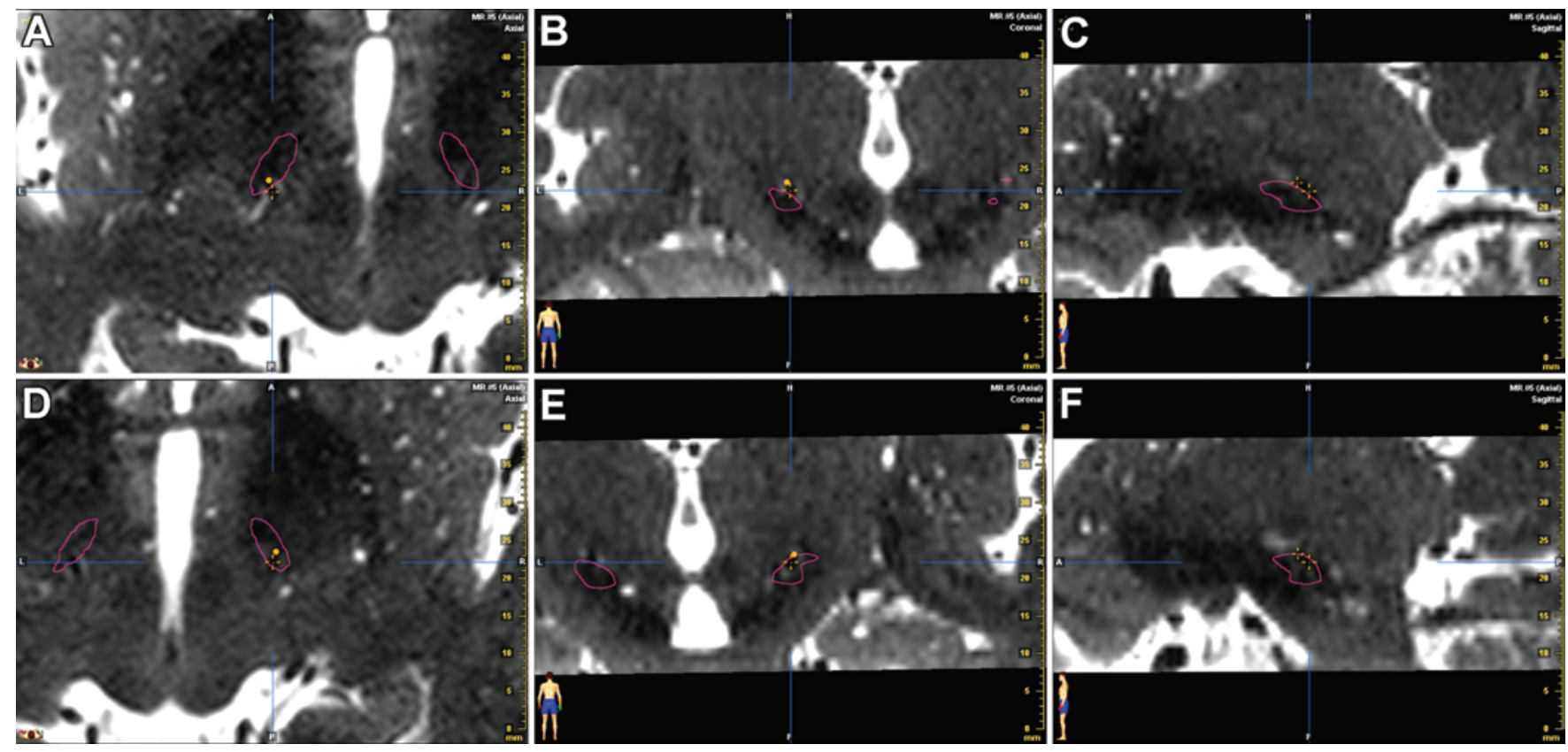

FIG. 1. Intraoperative T2-weighted MRI studies marked with preoperative manual segmentation of STN (pink). Panels A-C demonstrate left brain contacts and panels D-F demonstrate right brain contacts. A and $\mathbf{D}$ are axial, $\mathbf{B}$ and $\mathbf{E}$ are orthogonal, and $\mathbf{C}$ and $\mathbf{F}$ are sagittal slices through the deeper active contact (yellow crosshairs), with the more superficial interleaved active contact (yellow circles) at the cZl.

PD, but the effect may not be specific to this brain region or underlying disease process. We have received 3 additional anecdotal reports of drowning in patients who had undergone unilateral lesioning and contralateral DBS of the ventrointermediate nucleus of the thalamus (1 patient) and globus pallidus (2 patients) for PD in Western Australia. There is an urgent need to further investigate the prevalence of swimming dysfunction and its safety implications in more patients undergoing DBS at all basal ganglia and thalamic targets. At present we advise patients undergoing DBS of this possibility regardless of the DBS target, and recommend that they should be supervised when first returning to swim after surgery or substantive programming sessions. This conservative and simple measure should prevent any drownings while more clinical evidence is acquired. If clinicians move toward DBS earlier in the course of PD, ${ }^{8}$ then the potential for drowning may increase if younger and more physically active patients are treated.

\section{TABLE 2. Motor examination scores: MDS-UPDRS-III}

\begin{tabular}{lcc}
\hline $\begin{array}{c}\text { Assessment Items on } \\
\text { MDS-UPDRS-III (subsection) }\end{array}$ & Stimulation OFF* & Stimulation ON* \\
\hline Head $(3.1,3.2)$ & 1 & 2 \\
\hline Rigidity $(3.3)$ & 6 & 0 \\
\hline Akinesia $(3.4-3.9,3.14)$ & 7 & 0 \\
\hline Gait/posture (3.10-3.13) & 0 & 0 \\
\hline Tremor (3.15-3.18) & 22 & 0 \\
\hline Total & 36 & 2 \\
\hline
\end{tabular}

* The patient did not tolerate dopaminergics, so assessments were performed without any dopaminergic medications. Higher scores on the UPDRS-III indicate increased severity of motor symptoms, up to a maximum score of 132 .
The functional outcome of bilateral DBS of the PSA was regarded as successful by the treating clinicians as well as the patient. Neurological examination performed by our most experienced movement disorder neurologist (R.S.) did not reveal any abnormalities in gait, cerebellar signs, dystonia, or spasticity despite his knowledge of the swimming difficulty at the time of assessment. The inability to swim may be a form of task-specific dystonia; dystonic side effects have already been described in some patients undergoing PSA stimulation for tremor. ${ }^{3}$ The ZI within the PSA is an important node both within the thalamocorticobasal ganglia and the cerebellothalamocortical circuits and their descending projections, and it binds motor behavior with environmental sensory input. ${ }^{4}$ Electrical stimulation of the PSA may result in context-related (i.e., task-specific) motor dysfunction. It may be that neurological examination needs to include more task-specific functional testing of swimming ability (and perhaps other athletic performance) tailored to an individual patient's planned lifestyle.

Epidemiological studies of movement disorder surgery and drowning, and studies of swimming abilities in patients undergoing DBS are now required. The basal ganglia are thought to play an important role in motor control, and are thought to modulate the initiation and execution of movements so that they are performed in a smooth and fluid manner. Given the effect of high-frequency stimulation of the PSA near the cZI in this patient, we hypothesize that the basal ganglia play an important role in the performance of athletic movements that involve motor learning, such as swimming. Exploration of the effects of DBS on other targets involving corticostriatal and corticosubthalamic afferents and the feedback circuits between the glo- 
bus pallidus interna and substantia nigra pars reticulata that modulate basal ganglia output ${ }^{5}$ may shed light on potential mechanisms of athletic dysfunction after functional neurosurgery. The combination of epidemiological and biomechanical studies will establish which patients may develop deficits affecting swimming specifically, and will advance our understanding of the basal ganglia and athletic performance generally.

\section{Acknowledgment}

We thank Mujeeb Khan for assistance in the editing and production of the video.

\section{References}

1. Deuschl G, Schade-Brittinger C, Krack P, Volkmann J, Schäfer H, Bötzel K, et al: A randomized trial of deep-brain stimulation for Parkinson's disease. N Engl J Med 355:896908, 2006

2. Follett KA, Weaver FM, Stern M, Hur K, Harris CL, Luo P, et al: Pallidal versus subthalamic deep-brain stimulation for Parkinson's disease. N Engl J Med 362:2077-2091, 2010

3. Fytagoridis A, Åström M, Wårdell K, Blomstedt P: Stimulation-induced side effects in the posterior subthalamic area: distribution, characteristics and visualization. Clin Neurol Neurosurg 115:65-71, 2013

4. Mitrofanis J: Some certainty for the "zone of uncertainty"? Exploring the function of the zona incerta. Neuroscience 130:1-15, 2005

5. Obeso JA, Rodríguez-Oroz MC, Benitez-Temino B, Blesa FJ, Guridi J, Marin C, et al: Functional organization of the basal ganglia: therapeutic implications for Parkinson's disease. Mov Disord 23 (Suppl 3):S548-S559, 2008

6. Patel NK, Plaha P, Gill SS: Magnetic resonance imaging-directed method for functional neurosurgery using implantable guide tubes. Neurosurgery 61 (5 Suppl 2):358-366, 2007

7. Plaha P, Ben-Shlomo Y, Patel NK, Gill SS: Stimulation of the caudal zona incerta is superior to stimulation of the subthalamic nucleus in improving contralateral parkinsonism. Brain 129:1732-1747, 2006

8. Schuepbach WM, Rau J, Knudsen K, Volkmann J, Krack
P, Timmermann L, et al: Neurostimulation for Parkinson's disease with early motor complications. N Engl J Med 368:610-622, 2013

9. Thani NB, Bala A, Lind CR: Accuracy of magnetic resonance imaging-directed frame-based stereotaxis. Neurosurgery 70 (1 Suppl Operative): 114-124, 2012

10. Weaver FM, Follett K, Stern M, Hur K, Harris C, Marks WJ Jr, et al: Bilateral deep brain stimulation vs best medical therapy for patients with advanced Parkinson disease: a randomized controlled trial. JAMA 301:63-73, 2009

11. Williams A, Gill S, Varma T, Jenkinson C, Quinn N, Mitchell R, et al: Deep brain stimulation plus best medical therapy versus best medical therapy alone for advanced Parkinson's disease (PD SURG trial): a randomised, open-label trial. Lancet Neurol 9:581-591, 2010

\section{Disclosure}

The authors report no conflict of interest concerning the materials or methods used in this study or the findings specified in this paper.

\section{Author Contributions}

Conception and design: Lind, Thorburn, Garcia-Vega, Starkstein. Acquisition of data: Bangash, Thorburn, Garcia-Vega, Walters. Analysis and interpretation of data: Lind, Bangash, Garcia-Vega, Stell. Drafting the article: Bangash. Critically revising the article: Lind, Bangash, Stell, Starkstein. Reviewed submitted version of manuscript: Lind, Thorburn, Garcia-Vega, Walters, Stell, Starkstein. Administrative/technical/material support: Walters. Study supervision: Lind.

\section{Supplemental Information Videos \\ Video 1. https://vimeo.com/141799222. \\ Correspondence}

Christopher Lind, Neurosurgical Service of Western Australia, 1st Fl., G Block, Sir Charles Gairdner Hospital, Hospital Ave., Nedlands WA 6009, Australia. email: christopher.lind@health. wa.gov.au. 